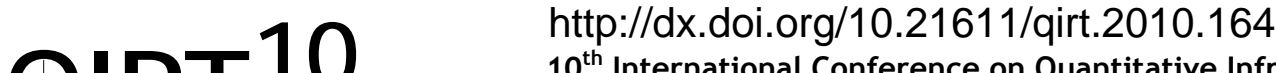 \\ $10^{\text {th }}$ International Conference on Quantitative InfraRed Thermography \\ July 27-30, 2010, Québec (Canada)
}

\section{Infrared Image Processing for Online Quality Control in Laser Welding}

\author{
by Samir Sahli*, Simon Fissette ${ }^{\star \star}$ and Xavier Maldague ${ }^{\star \star \star}$ \\ * Image Science group, Center for Optics, Photonics and Lasers, Université Laval, Québec (Québec), \\ Canada G1K 7P4, samir.sahli.1@ulaval.ca \\ **Centre Spécialisé de Technologie Physique du Québec Inc., Cégep de La Pocatière, La Pocatière (Québec), \\ Canada GOR 1Z0, sfissette@cegeplapocatiere.qc.ca \\ ***Elect. Eng. Dept., Université Laval, Québec (Quebec), Canada G1V 0A6, Xavier.Maldague@gel.ulaval.ca
}

\begin{abstract}
Laser welding is currently applied to various industries. Since traditional offline testing of the welds is costly and rather inefficient, online inspection systems are being developed to reduce cost and increase productivity while maintaining high welding quality. This paper presents a way of monitoring the weld quality using infrared thermography. An infrared camera was placed behind the laser head and captured the cooling of the weld. A lightweight image processing algorithm, fast enough to be run online, was proposed to extract relevant information from the images and thus used to monitor the penetration depth of the weld.
\end{abstract}

\section{Introduction}

In the field of sheet metal welding, laser welding is known as a highly reliable and efficient process. However, there are some typical faults such as seam irregularities that cannot be avoided completely. For this reason, industry has a need for systems which automatically detect faults and welding problems online to allow a fast response. Systems commercially found are usually based on the evaluation of the signals that are emitted from the welding process (at the laser keyhole where the metal is actually vaporized) rather than inspecting the weld itself [1]

Infrared thermography has been proposed in the past to monitor weld quality, but was shown to be technically difficult at the time $[2,3]$. Poor processing power, slow time response and low resolution of the infrared cameras were hold responsible. Few researchers also used single detectors or arrays to measure infrared radiation emitted behind the keyhole and used it as a monitoring tool with limited success $[4,5]$. In this work, we used an infrared camera to look behind the process where the weld is cooling down and emit a strong infrared signature to deduce properties of the weld.

\section{Experimental setup}

The experimental setup involve is quite simple. An infrared camera is attached on the laser head so that it records the cooling of the weld behind the laser keyhole. Different combinations of speed and laser power were used to weld 304 stainless in a simple bead on plate setup. The camera used is a Phoenix from Indigo Systems which is sensitive in 8-9.2 $\mu$ m range and has $640 \times 512$ pixels and a 25mm lens. Another magnifying lens was added with a focal length of $127 \mathrm{~mm}$ giving the images a $100 \mu \mathrm{m} /$ pixel resolution. The length along the weld the camera sees was about $6 \mathrm{~cm}$.

\section{Image processing}

In order to monitor the laser welding, we extracted from the Infrared image (Fig. 1) the Full-Width Half-Maximum (FWHM) and then the Surface Density at different delays of time after the keyhole along the weld. A lightweight robust algorithm has been proposed to take into account the real-time needs and also constraints inherent in the chain of production (e.g., splatter welding, velocity of the weld robot).

As shown in the Fig. 1 the weld image obtained with the IR camera is quite symmetric. We exploited this property to detect automatically the center of the weld, frame by frame, by computing an edge map with the Canny edge detector [6]. From the binary edge map obtained, we determined the location of the equidistant point between the upper and lower pixel of the each column for a given frame. The pixels retained are considered as belonging to the symmetry axis. Hence, a linear regression was computed taking the pixels from the previous step in order to define the equation of the center weld. A temporal scale, that represented the elapsed time Ti after the keyhole, was associated to the center weld according to the Robot Laser Welding Speed, $V$ and the camera frame rate $\tau$.

The FWHM value monitoring is done in real-time by computing a scan line at an appropriated location on the IR image that corresponded to the time Ti. Regarding the surface density, it was estimated from a Region of Interest (ROI), as 
shown by the pink bounding box in Fig. 1 . This region is centered on the symmetry axis and located at the time Ti. The dimensions of the ROI correspond to the area $A=L \cdot \overline{F W H M}$ with $L=V / \tau$ and $\overline{F W H M}$ the mean value of the FWHM estimated from scan line of each column belonging to the Region of Interest.

Robot Laser Welding Speed: $40 \mathrm{~mm} / \mathrm{s}$ observed at $\mathrm{Ti}=400 \mathrm{~ms}$ after the keyhole

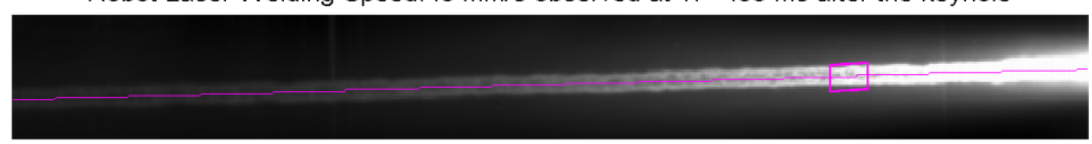

Full-Width Half-Maximum (FWHM), mean value : 1.1429 [mm]

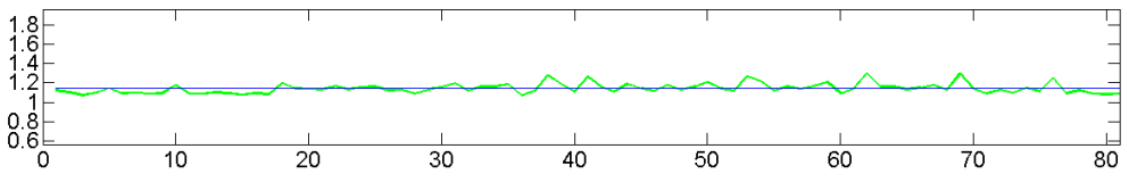

Surface Density, mean value : $1.095 \mathrm{E}+006\left[\right.$ Energy $\left./ \mathrm{mm}^{2}\right]$

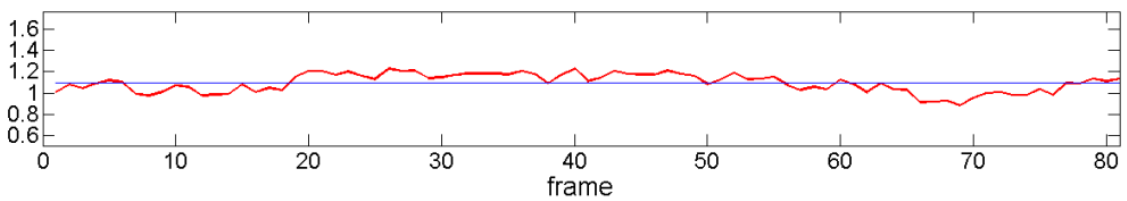

Fig. 1 : From top to bottom, real-time center weld detection (pink line) and ROI (the pink bounding box) for a time Ti=400ms after the keyhole, the FWHM and the Surface Density value monitored for each frame from the Infrared camera.

\section{Stability and evaluation of the penetration depth}

To monitor the penetration depth, we use the bottom image in Fig. 1 which shows the surface density of radiated energy by a given $\mathrm{ROI}(\mathrm{Ti}=400 \mathrm{~ms}$ ) in function of the frame number. Each frame corresponds to a physical area on the plate knowing $V$ and $\tau$. Using different combinations of welding speed and laser power, we varied the penetration depth and then correlated it to the aforementioned radiated energy. Then the penetration depth can be monitored along the weld in realtime with the proposed algorithm. Furthermore, many ROIs associated with a given time behind the keyhole can be measured and correlated to a penetration depth and strengthen the evaluation. Using many ROls can also be used to measure the cooling rate which could give an additional tool to detect anomalies.

\section{Conclusion}

We demonstrated the possibility of using infrared thermography and subsequent image processing to measure and monitor in real-time the penetration depth during laser welding.

\section{REFERENCES}

[1] Shao J. and Yan Y, "Review of techniques for On-line Monitoring and Inspection of Laser Welding", Journal of Physics: Conference Series 15 (2005) 101-107

[2] Nagarajan S. and Chin B.A., "Infrared Techniques for Real-Time Weld Quality Control", Infrared methodology and technology, Chapter X (1995) 367-396

[3] Tönshoff H.K., Alvensleben F.V., Ostendorf A. and Hillers O., M. Stailmach, "Online quality control of laser welding using supporting line-shaped IR-detection", Conference on Sensors and Controls for Intelligent Machining II, SPIE Vol. 3832, (1999) 0277-786

[4] Doong J.L., Wu C.-S. and Hwang J.-R., "Infrared Temperature Sensing of Laser Welding", Int. J. Mach. Tools Manufact. Vol. 31, No. 4, (1991) pp. 607-616

[5] Lee M.-Y. and Kim J.-W., "On-Line Penetration Depth Measurement System Using Infrared Temperature Sensing on Co2 Laser Welding", Key Engineering Materials Vols. 270-273 (2004) pp 2308-2314

[6] Canny, John, "A Computational Approach to Edge Detection," IEEE Transactions on Pattern Analysis and Machine Intelligence,Vol. PAMI-8, No. 6, 1986, pp. 679-698. 